\title{
Fasudil reduces monocrotaline-induced pulmonary arterial hypertension: comparison with bosentan and sildenafil
}

\author{
K.T.B. Mouchaers*, I. Schalij*, M.A. de Boer*, P.E. Postmus*, \\ V.W.M. van Hinsbergh", G.P. van Nieuw Amerongen", \\ A. Vonk Noordegraaf* and W.J. van der Laarse ${ }^{\#}$
}

ABSTRACT: Pulmonary arterial hypertension (PAH) still cannot be cured, warranting the search for novel treatments.

Fasudil (a Rho kinase inhibitor) was compared with bosentan (an endothelin receptor blocker) and sildenafil (a phosphodiesterase 5 inhibitor), with emphasis on right ventricular (RV) function, in a reversal rat model of monocrotaline (MCT)-induced PAH. In addition, the effects of combining bosentan or sildenafil with fasudil were studied.

MCT (40 $\mathrm{mg} \cdot \mathrm{kg}$ body weight ${ }^{-1}$ ) induced clear PAH in male Wistar rats $(\mathrm{n}=9)$. After 28 days, echocardiography, RV catheterisation and histochemistry showed that cardiac frequency, stroke volume and RV contractility had deteriorated, accompanied by RV dilatation and hypertrophy, and marked pulmonary arterial wall thickening. Mean pulmonary arterial pressure and pulmonary vascular resistance increased significantly compared to healthy rats $(n=9)$. After 14 days, MCTtreated rats received a 14-day oral treatment with bosentan, sildenafil, fasudil or a combination of fasudil with either bosentan or sildenafil (all $n=9$ ). All treatments preserved cardiac frequency, stroke volume and RV contractility, and reduced pulmonary vascular resistance and RV dilatation. Fasudil lowered RV systolic pressure and mean pulmonary arterial pressure significantly, by reducing pulmonary arterial remodelling, which reduced $\mathrm{RV}$ hypertrophy. Combining bosentan or sildenafil with fasudil had no synergistic effect.

Fasudil significantly improved PAH, to a greater degree than did bosentan and sildenafil.

KEYWORDS: Bosentan, combination drug therapy, fasudil, pulmonary hypertension, right ventricle, sildenafil

diopathic pulmonary arterial hypertension $(\mathrm{PAH})$ is a severe, progressive and fatal disease, which is characterised by increased pulmonary vascular resistance (PVR), caused by extensive remodelling of the vasculature of the lungs. The right ventricle (RV) of the heart initially adapts by hypertrophy to maintain cardiac output, but eventually fails $[1,2]$. Various treatments for $\mathrm{PAH}$ have been developed over time, including anticoagulant therapy, prostacyclin infusion, endothelin (ET) receptor (ETR) blockade and phosphodiesterase (PDE) 5 inhibition, either alone or in combination therapy $[3,4]$. Despite several treatment options, the disease remains incurable and the prognosis grim, warranting the search for novel medications.
A potential candidate for treatment is the Rho kinase inhibitor fasudil [5, 6]. Various studies have shown that Rho kinase, a potent effector of the small GTPase RhoA (ras homologue gene family, member A), is actively involved in the pathogenesis of $\mathrm{PAH}[6,7]$. Activation of RhoA/Rho kinase occurs in both endothelial cells (ECs) and vascular smooth muscle cells (SMCs), in which it causes structural cytoskeletal changes, leading to altered cell adhesion properties and hypercontraction [8, 9]. Inhibition of Rho kinase exerts both acute and prolonged vasodilatory effects on the pulmonary arteries, and also reduces structural remodelling by inhibiting proliferation of SMCs and fibroblasts [10].

For editorial comments see page 709.

This article has supplementary material accessible from www.erj.ersjournals.com

AFFILIATIONS

*Dept of Pulmonary Diseases, and \#Laboratory for Physiology, Institute for Cardiovascular Research, Vrije Universiteit Medical Center, Amsterdam, The Netherlands.

\section{CORRESPONDENCE}

A. Vonk Noordegraaf Dept of Pulmonary Diseases Vrije Universiteit Medical Center P.0. Box 7057

1007 MB

Amsterdam

The Netherlands

E-mail: a.vonk@vumc.nl

Received:

Aug 162009

Accepted after revision:

Feb 122010

First published online:

March 292010 
Beneficial effects of Rho kinase inhibition have been observed in various cardiovascular disorders [5]. However, potential adverse effects may also be envisaged. RhoA is essential for morphogenesis of cardiomyocytes during the development of the heart [11, 12]. A large part of the developmental gene programme is reactivated in hypertrophied myocardium [1315], and evidence is increasing that RhoA is an important mediator of the hypertrophic response [16, 17]. Inhibition of Rho kinase might, therefore, harm the adaptive response of the $\mathrm{RV}$ to pressure overload. In view of the significant importance of the RV in coping with the increased load, it is crucial to gain an insight into the effects of Rho kinase inhibition on RV function and adaptation to PAH.

Therefore, the effects of Rho kinase inhibition were investigated in a treatment model of monocrotaline (MCT)-induced $\mathrm{PAH}$ in the rat, with emphasis on RV function, and the results were compared with those of two of the most commonly used therapies in PAH, namely ETR blockade by bosentan and PDE5 inhibition by sildenafil. In addition, it was investigated whether combination of fasudil with bosentan or sildenafil was more effective than the various monotherapies.

\section{MATERIALS AND METHODS}

Additional information regarding methods and reagents is provided in the online supplementary material.

\section{Study design}

PAH was induced in 63 rats by a single injection of MCT (SigmaAldrich, Zwijndrecht, the Netherlands; $40 \mathrm{mg} \cdot \mathrm{kg}_{\text {body weight }}{ }^{-1}$ subcutaneously; body mass 180-200 g). This dose was selected because it leads to severe $\mathrm{PAH}$ and mortality during the first month after administration is low [18], permitting similar treatment periods in all groups. At 14 days after MCT injection, when RV systolic pressure (RVSP) in MCT-treated rats had increased from $25 \pm 2$ to $49 \pm 10 \mathrm{mmHg}$ [16], the rats randomly received oral treatment with bosentan (Actelion Pharmaceuticals, Allschwil, Switzerland; $300 \mathrm{mg} \cdot \mathrm{kg}$ body weight ${ }^{-1} \cdot$ day $^{-1} ; \mathrm{n}=9$ ), sildenafil (Pfizer, Inc., New York, NY, USA; $10 \mathrm{mg} \cdot \mathrm{kg}$ body weight $^{-1} \cdot$ day $^{-1} ; \mathrm{n}=9$ ), fasudil (Mercachem, Nijmegen, the Netherlands; $100 \mathrm{mg} \cdot \mathrm{kg}$ body weight $^{-1} \cdot$ day $^{-1} ; \mathrm{n}=9$ ) or the combinations fasudil plus bosentan (same dosages; $n=9$ ) or fasudil plus sildenafil (same dosages; $n=9$ ). All of the rats were trained to eat vanilla pudding, which served as vehicle for sildenafil and bosentan [18]. Fasudil was administered by gavage. Untreated healthy rats served as reference (control) for PAH rats $(n=9)$. All experiments were approved by the Institutional Animal Care and Use Committee of the Institute for Cardiovascular Research (Free University University Medical Center, Amsterdam, the Netherlands) and conform to the Helsinki convention for the use and care of animals.

\section{Echocardiographic and haemodynamic measurements}

At days 0,14 and 28, rats were anaesthetised (2.0\% isoflurane in 1:1 oxygen/air mix; Pharmachemie, Haarlem, the Netherlands), as previously described [4, 18]. Echocardiography was performed before administration of the drugs (i.e. $24 \mathrm{~h}$ after the previous dose) in order to study general cardiac and RV-specific function, using an Aloka SSD4000 ultrasonographic system (Biomedic, Almere, the Netherlands). The following parameters were determined: cardiac frequency, stroke volume (SV) and cardiac output (CO) as general measures of cardiac function; RV end-systolic (RVESD) and end-diastolic diameter (RVEDD) as measures of RV dilatation; and RV fractional shortening (RVFS) and tricuspid annular plane systolic excursion (TAPSE) as measures of RV contractility [4, 16-19]. Analyses were performed offline (Image-Arena 2.9.1; TomTec Imaging Systems, Unterschleissheim, Germany). RVSP was measured and mean pulmonary arterial pressure $\left(\bar{P}_{\text {pa }}\right)$ determined as previously described [4, 20-22]. Data were
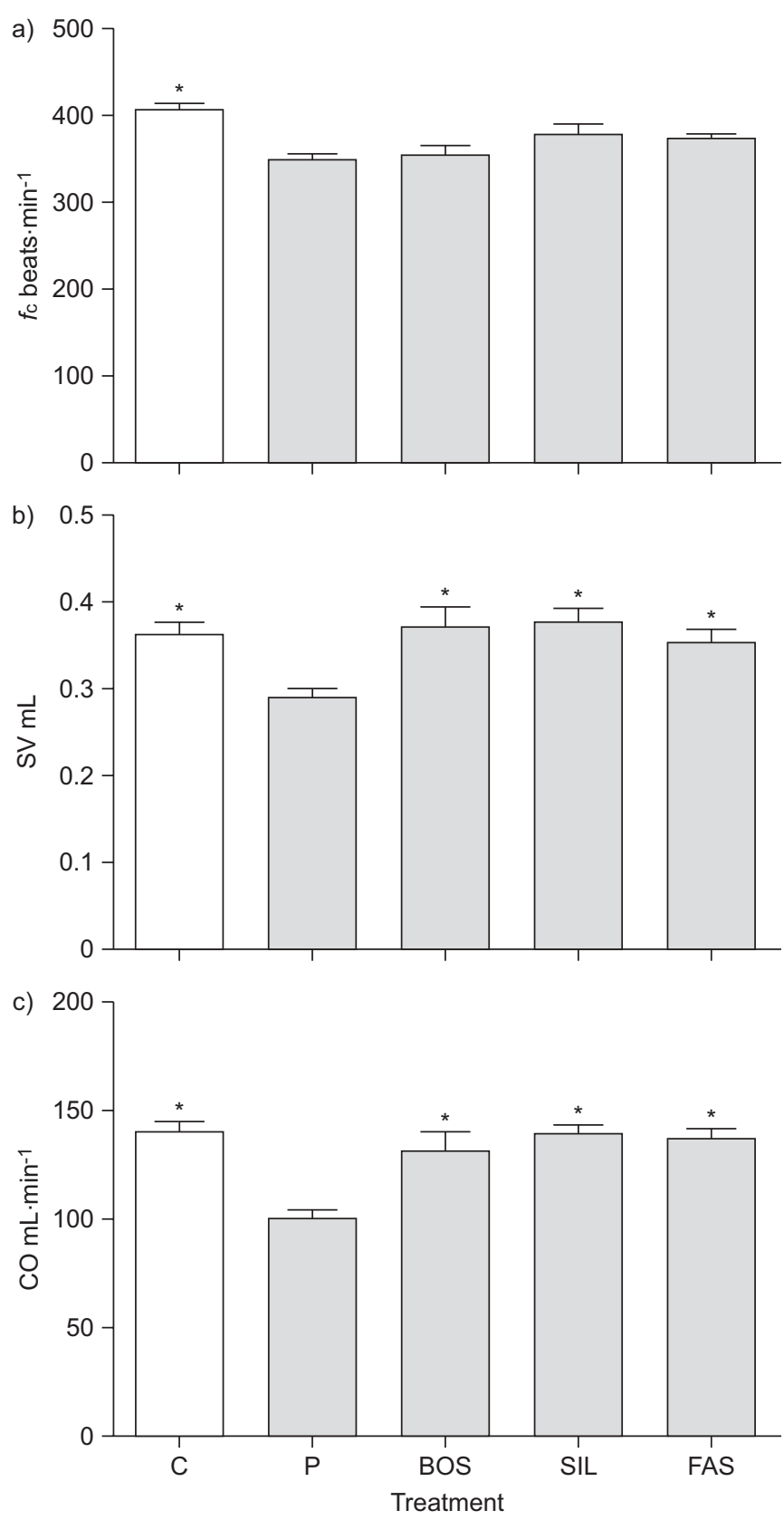

FIGURE 1. General echocardiographic cardiac function parameters. Monocrotaline $(\mathrm{MCT} ;-)$ induced significant pulmonary arterial hypertension, with signs of cardiac dysfunction, indicated by a lower cardiac frequency ( $\left.f_{c} ; a\right)$, and reduced stroke volume (SV; b) and cardiac output (CO; c) compared to control (C: $\square)$. Treatment of MCT-treated rats with fasudil (FAS), bosentan (BOS) and sildenafil (SIL) preserved SV and CO. Data are presented as mean \pm SEM $(n=9)$. P: placebo. *: $p<0.05$ versus $P$. 
obtained using a PowerLab setup (ADinstruments, Bella Vista, Australia). Systemic blood pressure was monitored using a fluid-filled catheter inserted into the left carotid artery. PVR was determined as previously described [4, 20, 22].

\section{Morphometric analysis of the lungs}

After excision, lungs were fixed in $4 \%$ formaldehyde in PBS. Pulmonary sections $(5 \mu \mathrm{m})$ were stained with Elastica-van Gieson stain for morphometric analysis of vascular dimensions, as previously described [23, 24]. Minimally 40 transversely cut pulmonary arterioles, randomly distributed over the lungs and with an outer diameter ranging 25-200 $\mu \mathrm{m}$, were measured, using ImageJ image analysis software (ImageJ for Windows 1.41; National Institutes of Health, Bethesda, MD, USA). Subsequently, relative pulmonary arterial wall thickness (PAWT) was calculated as follows [23]:

\section{PAWT $=2 \times$ medial wall thickness/external diameter}

\section{RV hypertrophy}

After sacrifice, hearts were excised and the mass ratio between the RV and the left ventricle (LV) plus septum was calculated as a measure of RV hypertrophy. In order to study individual cardiomyocyte hypertrophy, cryosections of myocardial tissue $(5 \mu \mathrm{m})$ were stained using haematoxylin-eosin and the cross-sectional area (CSA) of 40 cardiomyocytes was measured randomly in the RV, as previously described [18].

\section{Statistical analysis}

Data are presented as mean \pm SEM. In order to determine differences between groups, ANOVA was used with Dunnett's correction for multiple comparisons with a single control group. In the present study, untreated MCT-treated rats served as control for MCT-treated rats that received fasudil, bosentan or sildenafil. In order to determine differences between untreated MCT-treated and healthy control rats, an unpaired t-test was used. Differences between fasudil treatment and the combination of fasudil and bosentan or of fasudil and sildenafil were evaluated by ANOVA with Bonferroni correction. A p-value of $<0.05$ was considered significant.

\section{RESULTS}

Additional data are provided in the online supplementary material.
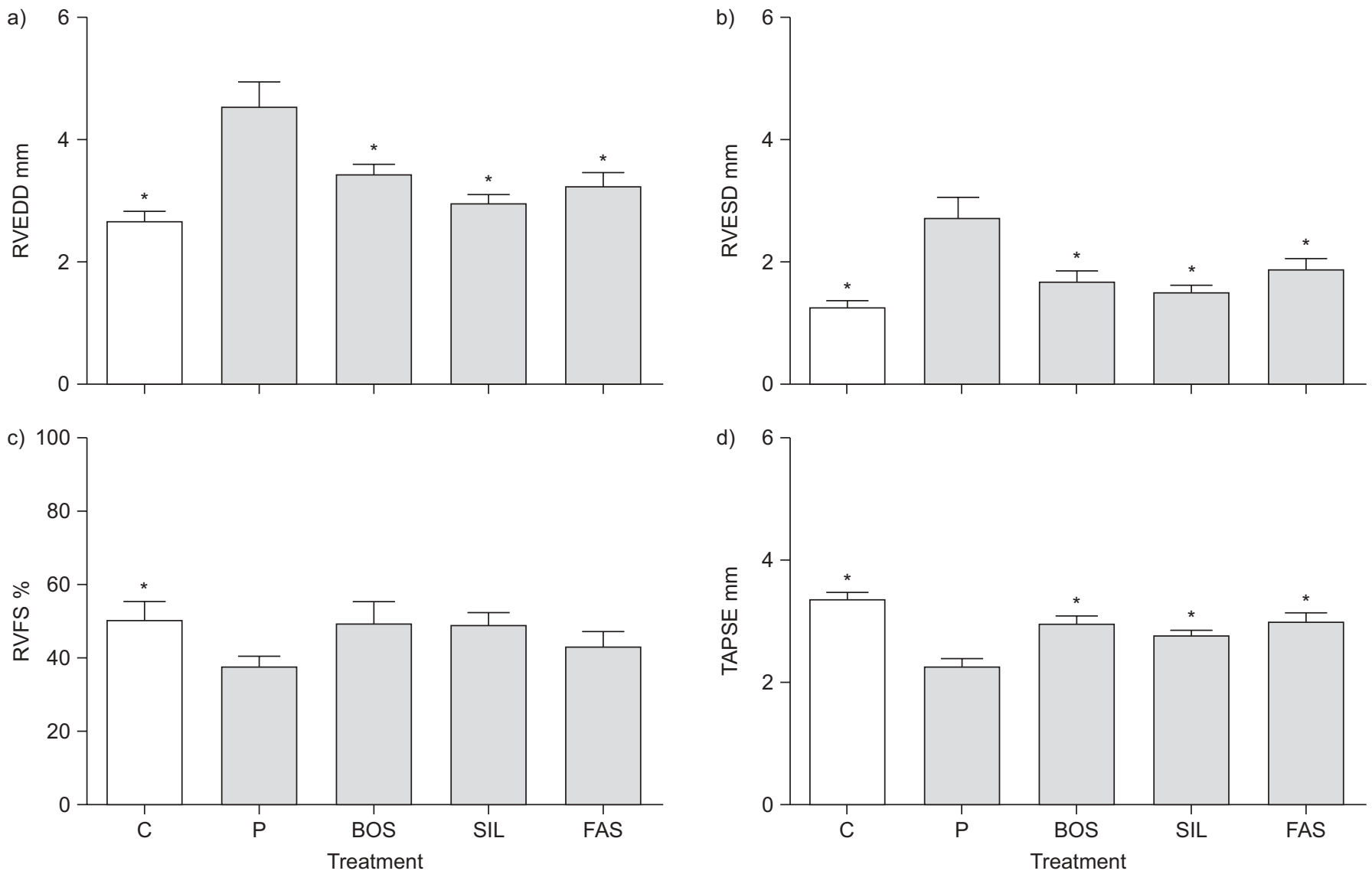

FIGURE 2. Echocardiography was used to study right ventricular (RV) dimensions and RV-specific function. Monocrotaline ( $\square$ ) induced marked dilatation of the RV (a and b). In addition, RV contractility was reduced, as indicated by reduced RV fractional shortening (RVFS) (c) and reduced tricuspid annular plane systolic excursion (TAPSE) (d). All three treatments significantly reduced RV dilatation. The preservation of RV contractility was confirmed by TAPSE. Data are presented as mean \pm SEM ( $n=9$ ). RVESD: right ventricular end-systolic diameter; RVEDD: right ventricular end-diastolic diameter; C: control ( $\square$ ); P: placebo; BOS: bosentan; SIL: sildenafil; FAS: fasudil. *: $p<0.05$ versus $P$. 


\section{Cardiac function and RV contractility using echocardiography}

An extensive echocardiographic protocol was performed in order to study cardiac function. On days 0 and 14, no differences were found between groups in cardiac function (data not shown). On day 28, MCT-treated rats showed a reduction in cardiac frequency compared to control animals under similar anaesthetic conditions, but no differences were found between untreated MCT-treated rats and their bosentan-, sildenafil- or fasudil-treated counterparts (fig. 1a). SV and CO were significantly reduced in MCT-treated rats compared to control, and were restored by all three treatments (fig. $1 \mathrm{~b}$ and $\mathrm{c}$ ).

Subsequently, the RV was specifically studied with regard to its dimensions and contractility. MCT induced severe dilatation of the RV, as indicated by increased RVEDD and RVESD (fig. 2a and b). This resulted in a decrease in RVFS (fig. 2c). All three treatments reduced RV dilatation in MCT-treated rats (fig. 2a and b). Although RVFS in the treated groups was similar to that in control rats, its difference from that of untreated MCT rats did not reach significance. TAPSE was determined as a specific measure of RV contractility and proved to be significantly lower in untreated MCT-treated rats compared to control (fig. 2d). In MCT-treated rats, all treatments significantly improved RV contractility according to TAPSE.
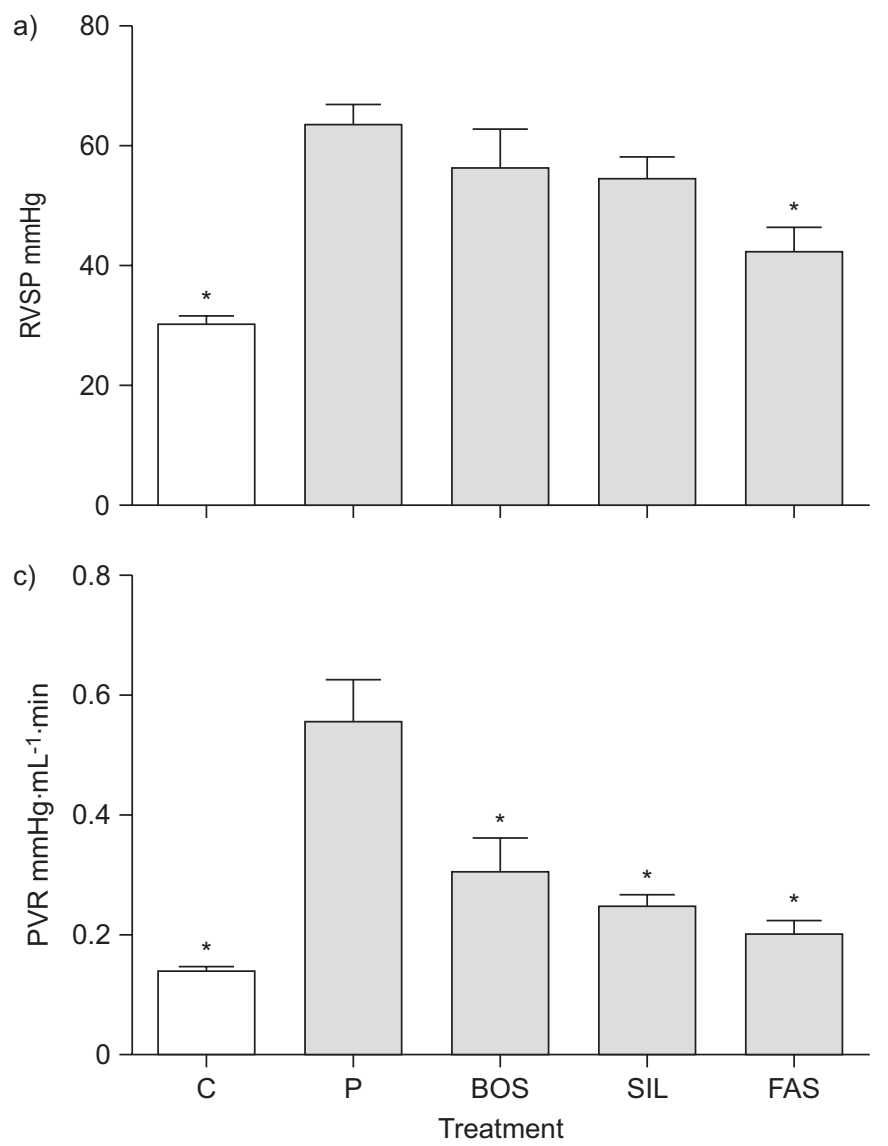

\section{Haemodynamics}

After echocardiography on day 28, right heart catheterisation was performed to determine the severity of PAH. RVSP was measured, and $\bar{P}$ pa and PVR determined, all of which were significantly increased in untreated MCT-treated rats versus control (fig. 3a-c). Rho kinase inhibition by fasudil reduced these parameters significantly in MCT-treated rats, although a trend towards a lower RVSP and $\bar{P}$ pa was caused by the single treatments with ETR antagonism by bosentan and PDE5 inhibition by sildenafil. Despite the absence of a significant effect of bosentan and sildenafil on afterload, PVR was reduced by all treatments. It should be noted that the strongest effect on PVR was seen upon fasudil treatment. None of the treatments had an effect on the systemic circulation, as indicated by similar systemic pressures in all groups (fig. 3d).

\section{Remodelling of pulmonary arteries}

It was subsequently evaluated whether or not the treatments affected the remodelling of the pulmonary arterial wall. Morphometric analysis was performed on small pulmonary arteries, and relative wall thickness (PAWT) measured. MCT induced severe wall thickening of the pulmonary arteries, as indicated by an increase in PAWT (fig. 4). Only fasudil treatment was able to prevent or lessen such remodelling to a significant extent.
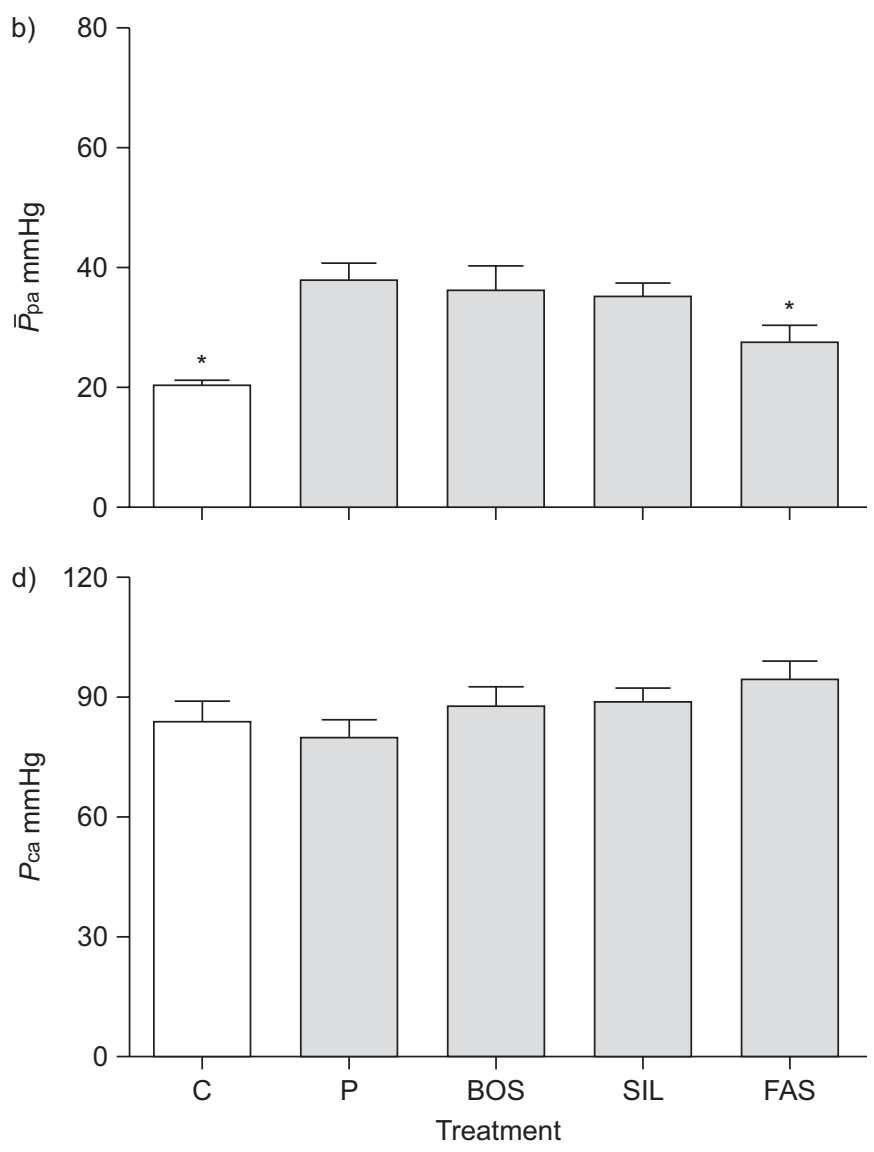

FIGURE 3. Right ventricular (RV) catheterisation was performed to measure the severity of pulmonary arterial hypertension (PAH). Monocrotaline (MCT; $\square$ ) significantly induced PAH, as indicated by a marked increase in RV systolic pressure (RVSP; a), mean pulmonary arterial pressure (Ppa; b) and pulmonary vascular resistance (PVR; C). MCT did not affect systemic pressure as measured in the carotid artery $\left(P_{c a} ; d\right)$. Data are presented as mean \pm SEM $(n=9)$. C: control $(\square)$; P: placebo; BOS: bosentan; SIL: sildenafil; FAS: fasudil. *: $p<0.05$ versus $P$. 

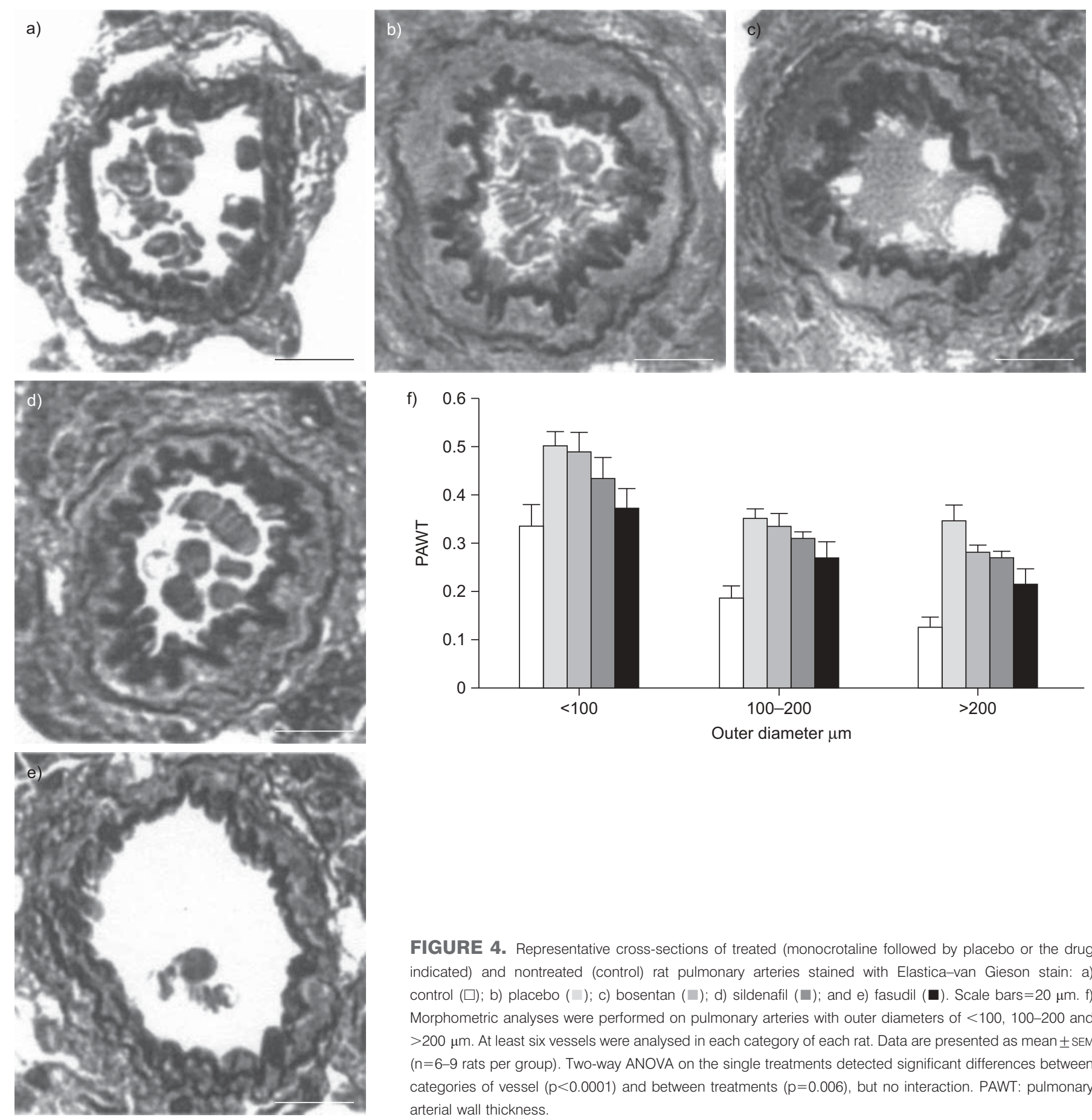

FIGURE 4. Representative cross-sections of treated (monocrotaline followed by placebo or the drug indicated) and nontreated (control) rat pulmonary arteries stained with Elastica-van Gieson stain: a) control $(\square)$; b) placebo $(\square)$; c) bosentan ( $\square$ ); d) sildenafil $(\square)$; and e) fasudil ( $\square$ ). Scale bars=20 $\mu \mathrm{m}$. f) Morphometric analyses were performed on pulmonary arteries with outer diameters of $<100,100-200$ and $>200 \mu \mathrm{m}$. At least six vessels were analysed in each category of each rat. Data are presented as mean \pm SEM ( $n=6-9$ rats per group). Two-way ANOVA on the single treatments detected significant differences between categories of vessel $(p<0.0001)$ and between treatments $(p=0.006)$, but no interaction. PAWT: pulmonary arterial wall thickness.

\section{RV hypertrophy}

The degree of RV hypertrophy was determined as a reflection of $\mathrm{RV}$ adaptation to $\mathrm{PAH}$, by both calculating the ratio between the RV and the LV plus septum and measuring the CSA of individual RV cardiomyocytes. MCT induced severe RV hypertrophy, as indicated by an increase in the ratio of the RV to the LV plus septum and an increase in the CSA of individual RV cardiomyocytes (fig. 5). Fasudil markedly reduced RV hypertrophy, whereas bosentan and sildenafil had no significant effect. Further detailed data on mitochondrial content, myoglobin concentration and capillary density are presented in the online supplementary material.

\section{Effects of combination treatment on cardiac function, haemodynamics, pulmonary arterial remodelling and RV hypertrophy}

Subsequently, it was investigated whether or not combined treatment with fasudil and bosentan or sildenafil had additional ameliorating effects on PAH development (table 1). No significant further improvements from fasudil treatment of 

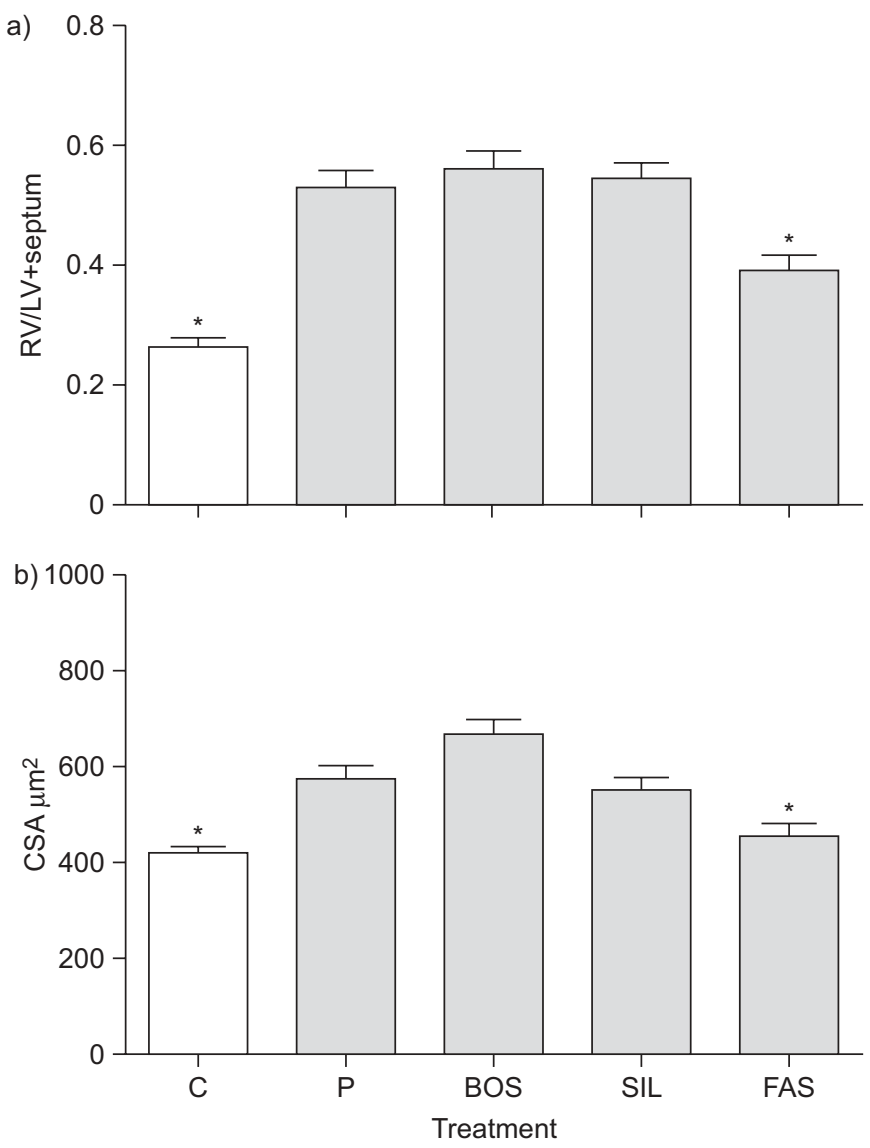

FIGURE 5. The degree of right ventricular (RV) hypertrophy was measured by: a) calculating the ratio between the RV and the left ventricle (LV) plus septum; and b) measuring the cross-sectional area (CSA) of individual RV cardiomyocytes. Data are presented as mean \pm SEM $(n=9)$. The pulmonary arterial hypertension-inducing effect of monocrotaline (MCT; $\square$ ) was clear from a marked increase in RV hypertrophy, on both the total ventricular and the individual cardiomyocyte level. Only fasudil reduced RV hypertrophy significantly in MCT-treated rats, whereas bosentan and sildenafil had no effect. C: control ( $\square$ ); P: placebo; BOS: bosentan; SIL: sildenafil; FAS: fasudil. *: $p<0.05$ versus $P$.

MCT-treated rats were found in rats treated with a combination of fasudil plus bosentan or fasudil plus sildenafil, or between the two combination groups as regards general echocardiographic parameters or RV-specific PAH parameters. Pulmonary vascular remodelling was not further reduced when bosentan or sildenafil were combined with fasudil. In addition, no differences were observed between single or combination treatment in RV hypertrophy, as indicated by similar RV/LV plus septum ratios and RV cardiomyocyte CSAs (table 1).

\section{DISCUSSION}

The main finding of the present study was that Rho kinase inhibition by fasudil is able to prevent disease progression in a treatment set-up of MCT-induced PAH, with preservation of RV function. This was associated with a significant reduction in RV afterload, accompanied by a marked reduction in pulmonary arterial wall thickening, which was not seen upon ETR blockade by bosentan or PDE5 inhibition by sildenafil.
From previous work, it is known that, in this PAH model, RVSP is already elevated at day 14 [16]. We specifically chose to study treatment of PAH that had already developed rather than a prevention set-up, and examined the effects of 14 days of treatment with bosentan and sildenafil, two drugs that are currently first-line treatments in PAH. Cardiac function was studied noninvasively, using echocardiography, and the degree of PAH was measured by right heart catheterisation. The present results as regards SV, CO and PVR are in line with previous studies using different methodologies, showing similar changes upon RV pressure overload in rat models of PAH [4, 19, 20, 23].

To our knowledge, this is the first study to directly compare Rho kinase inhibition with ETR blockade and PDE5 inhibition in a model of severe PAH. Although all treatments reduced PVR, fasudil was the only treatment that lowered $\bar{P}_{\text {pa. This was }}$ reflected in a marked reduction in RV dilatation and preserved RV contractility. The strongest effect on afterload was seen upon fasudil treatment, resulting in a marked reduction in RV hypertrophy, not observed in bosentan- or sildenafil-treated rats. The present findings confirm, in part, the beneficial effects on RV afterload of the current previously described medications [10, 25-27]. However, bosentan and sildenafil were more effective in these previous studies than in the present one, whereas the effects of fasudil were similar. One explanation might be that relatively young rats were subjected to $40 \mathrm{mg} \cdot \mathrm{kg}$ body weight ${ }^{-1} \mathrm{MCT}$, which may have caused a milder form of PAH. This may have precluded significant effects of bosentan and sildenafil on $\bar{P}_{\text {pa }}$ and RV hypertrophy. However, other studies with sildenafil have also shown marginal effects as regards reduction in $\mathrm{PAH}$ and $\mathrm{RV}$ remodelling in the pressure overloaded rat heart, with a preservation of RV function [28].

Morphometric analyses on pulmonary arteries strengthened the haemodynamic findings in the present study. A significant reduction in MCT-induced pulmonary arterial wall remodelling was observed upon fasudil treatment, but no significant improvements were found following bosentan or sildenafil treatment. As shown by other investigators, induction of apoptosis and reduction of proliferation of SMCs and fibroblasts can contribute to the attenuation of pulmonary arterial wall remodelling PAWT upon fasudil treatment [10, 29]. In addition, direct vasodilatory [30] and anti-inflammatory [10] effects of fasudil are probably involved in these positive haemodynamics.

Unfortunately, not all PAH patients benefit optimally from the available therapies. Therefore, the use of combination therapy is increasingly being applied in the treatment of PAH patients. Recent studies indicate that a combination of bosentan and sildenafil can be beneficial [31, 32]. In a recent study, a combination of fasudil and beraprost was more beneficial than the respective single treatments [29], but the combination of fasudil with the more commonly used PAH medications, such as bosentan and sildenafil, has not been examined. Under the present experimental MCT-induced conditions, these latter combination treatments had no synergistic effects. This finding is in agreement with the view of OKA et al. [33], reviewing the role of RhoA/Rho kinase signalling in many different models of PAH, who suggested that Rho kinase may serve as a point of convergence for the known pathogenic pathways involved, 


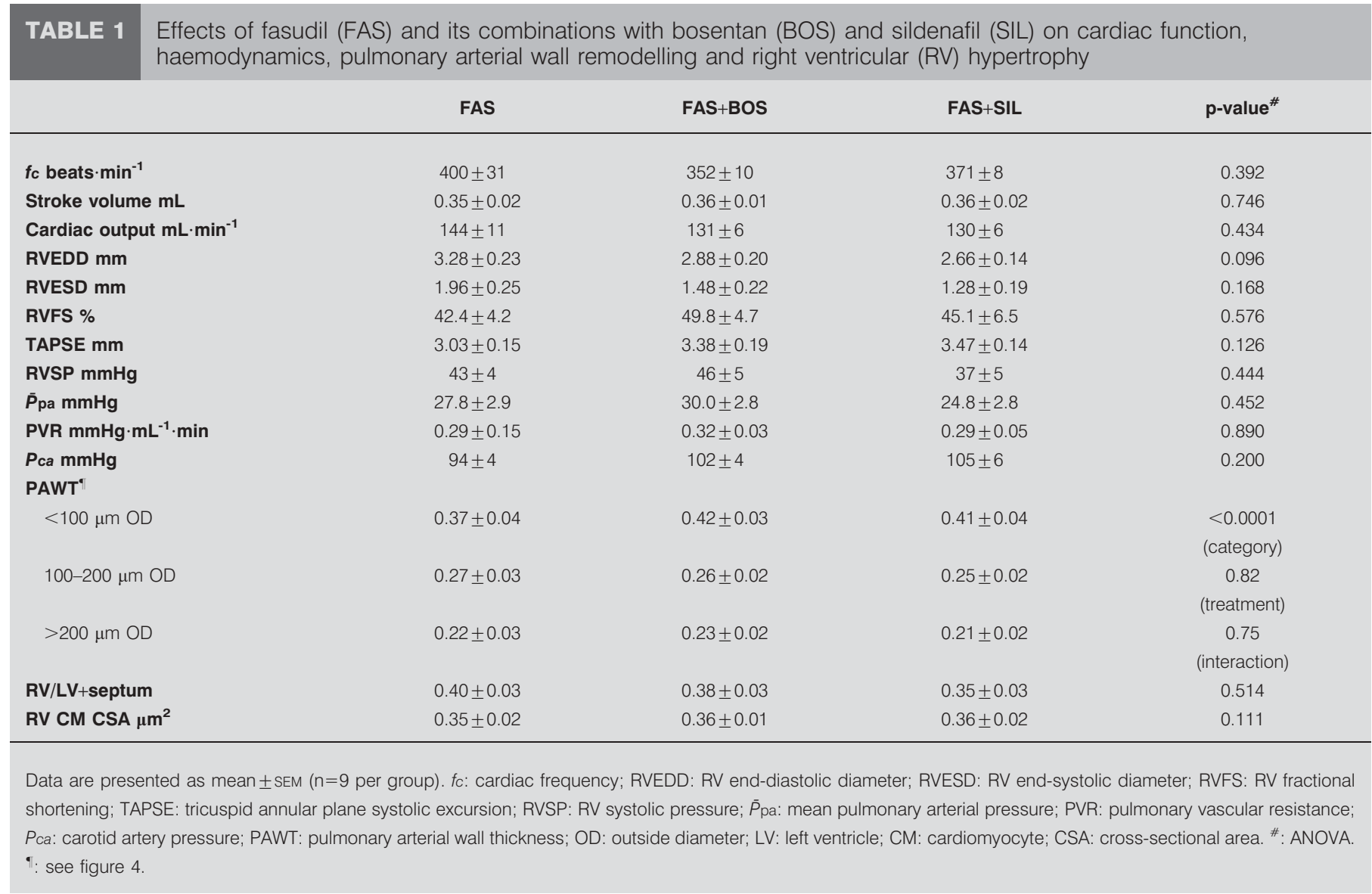

regardless of the cause of the PAH. In this view, bosentan or sildenafil might have limited additional effects to the actions of Rho kinase inhibition itself. ET-1 is a well-known activator of Rho kinase in $\mathrm{PAH}$, which might explain why bosentan has no additive effect [10, 34], and fasudil is known to restore downregulated endothelial nitric oxide synthase expression and reduced cyclic guanosine monophosphate levels, which might explain why sildenafil has no additive effect [35].

The majority of studies on Rho kinase inhibition in cardiovascular disease models indicate cardioprotective effects [36, 37]. However, care should be taken when considering this treatment, since numerous embryonic developmental programmes, including RhoA activation, are reactivated in pressure-overloaded myocardium $[14,38,39]$. The RV is extremely important in $\mathrm{PAH}$ and its adaptive capacity is the most important determinant of disease outcome. It may, therefore, be postulated that the antiproliferative effects of Rho kinase inhibition may adversely affect RV adaptation to PAH. However, the present data suggest that the antiproliferative effects of fasudil are limited to the lungs, without affecting normal RV function. In addition, the vasodilatory effects of fasudil were also limited to the pulmonary circulation, since no effects were observed on systemic blood pressure. This confirms the safety of longer-term use of fasudil, as suggested earlier by SHIMOKAWA and coworkers [5, 10, 29].

In conclusion, the present study shows that Rho kinase inhibition by fasudil is able to reverse MCT-induced PAH in rats, by reducing pulmonary vascular remodelling without affecting normal RV function. Compared to ETR blockade by bosentan and PDE5 inhibition by sildenafil, fasudil was more effective in the present experimental setting. In addition, combination treatment using fasudil plus bosentan or sildenafil had no complementary effects, compared to those of fasudil alone. Further clinical research is warranted [7].

\section{SUPPORT STATEMENT}

This study was supported by the Institute for Cardiovascular Research (Vrije Universiteit University Medical Center, Amsterdam, the Netherlands). G.P. van Nieuw Amerongen was supported by the Netherlands Heart Foundation (grant T2003-0032), and A. Vonk Noordegraaf by the Netherlands Organisation for Scientific Research (VIDI 917.96.306) (both The Hague, the Netherlands).

\section{STATEMENT OF INTEREST}

None declared.

\section{ACKNOWLEDGEMENTS}

We would like to thank N. Westerhof (Dept of Physiology, Vrije Universiteit Medical Center, Amsterdam, the Netherlands) for expert advice and discussion.

\section{REFERENCES}

1 Voelkel NF, Quaife RA, Leinwand LA, et al. Right ventricular function and failure. Report of a National Heart, Lung, and Blood 
Institute working group on cellular and molecular mechanisms of right heart failure. Circulation 2006; 114: 1883-1891.

2 Chin KM, Rubin LJ. Pulmonary arterial hypertension. J Am Coll Cardiol 2008; 51: 1527-1538.

3 Badesch DB, Champion HC, Sanchez MA, et al. Diagnosis and assessment of pulmonary arterial hypertension. J Am Coll Cardiol 2009; 54: Suppl. 1, S55-S66.

4 Mouchaers KT, Schalij I, Versteilen AM, et al. Endothelin receptor blockade combined with phosphodiesterase- 5 inhibition increases right ventricular mitochondrial capacity in pulmonary arterial hypertension. Am J Physiol Heart Circ Physiol 2009; 297: H200-H207.

5 Shimokawa H, Rashid M. Development of Rho-kinase inhibitors for cardiovascular medicine. Trends Pharmacol Sci 2007; 28: 296-302.

6 Wojciak-Stothard B. New drug targets for pulmonary hypertension: Rho GTPases in pulmonary vascular remodelling. Postgrad Med J 2008; 84: 348-353.

7 Guilluy C, Eddahibi S, Agard C, et al. RhoA and Rho kinase activation in human pulmonary hypertension: role of 5-HT signaling. Am J Respir Crit Care Med 2009; 179: 1151-1158.

8 Nieuw Amerongen GP, van Hinsbergh VW. Endogenous RhoA inhibitor protects endothelial barrier. Circ Res 2007; 101: 7-9.

9 Homma N, Nagaoka T, Morio Y, et al. Endothelin-1 and serotonin are involved in activation of RhoA/Rho kinase signalling in chronically hypoxic hypertensive rat pulmonary circulation. J Cardiovasc Pharmacol 2007; 50: 697-702.

10 Abe K, Shimokawa H, Morikawa K, et al. Long-term treatment with a Rho-kinase inhibitor improves monocrotaline-induced fatal pulmonary hypertension in rats. Circ Res 2004; 94: 385-393.

11 Zhao Z, Rivkees SA. Rho-associated kinases play an essential role in cardiac morphogenesis and cardiomyocyte proliferation. Dev Dyn 2003; 226: 24-32.

12 Wei L, Imanaka-Yoshida K, Wang L, et al. Inhibition of Rho family GTPases by Rho GDP dissociation inhibitor disrupts cardiac morphogenesis and inhibits cardiomyocyte proliferation. Development 2002; 129: 1705-1714.

13 Ogawa E, Saito Y, Kuwahara K, et al. Fibronectin signaling stimulates BNP gene transcription by inhibiting neuron-restrictive silencer element-dependent repression. Cardiovasc Res 2002; 53: 451-459.

14 Houweling AC, van Borren MM, Moorman AF, et al. Expression and regulation of the atrial natriuretic factor encoding gene Nppa during development and disease. Cardiovasc Res 2005; 67: 583-593.

15 Akazawa H, Komuro I. Cardiac transcription factor Csx/Nkx2-5: its role in cardiac development and diseases. Pharmacol Ther 2005; 107: 252-268.

16 Molkentin JD, Dorn GW. Cytoplasmic signaling pathways that regulate cardiac hypertrophy. Annu Rev Physiol 2001; 63: 391-426.

17 Lezoualc'h F, Métrich M, Hmitou I, et al. Small GTP-binding proteins and their regulators in cardiac hypertrophy. J Mol Cell Cardiol 2008; 44: 623-632.

18 Henkens IR, Mouchaers KT, Vliegen HW, et al. Early changes in rat hearts with developing pulmonary arterial hypertension can be detected with 3-dimensional electrocardiography. Am J Physiol Heart Circ Physiol 2007; 293: H1300-H1307.

19 Hardziyenka M, Campian ME, Bruin-Bon HA, et al. Sequence of echocardiographic changes during development of right ventricular failure in rat. J Am Soc Echocardiogr 2006; 19: 1272-1279.

20 Handoko ML, de Man FS, Happe CM, et al. Opposite effects of training in rats with stable and progressive pulmonary hypertension. Circulation 2009; 120: 42-49.
21 Chemla D, Castelain V, Humbert M, et al. New formula for predicting mean pulmonary artery pressure using systolic pulmonary artery pressure. Chest 2004; 126: 1313-1317.

22 Handoko ML, Schalij I, Kramer K, et al. A refined radio-telemetry technique to monitor right ventricle or pulmonary artery pressures in rats: a useful tool in pulmonary hypertension research. Pflugers Arch 2008; 455: 951-959.

23 Wagenvoort CA, Wagenvoort N, Draulans-Noe Y. Reversibility of plexogenic pulmonary arteriopathy following banding of the pulmonary artery. J Thorac Cardiovasc Surg 1984; 87: 876-886.

24 Schermuly RT, Pullamsetti SS, Kwapiszewska G, et al. Phosphodiesterase 1 upregulation in pulmonary arterial hypertension: target for reverse-remodeling therapy. Circulation 2007; 115: 2331-2339.

25 Jungebluth P, Ostertag H, Macchiarini P. An experimental animal model of postobstructive pulmonary hypertension. I Surg Res 2008; 147: 75-78.

26 Schermuly RT, Kreisselmeier KP, Ghofrani HA, et al. Chronic sildenafil treatment inhibits monocrotaline-induced pulmonary hypertension in rats. Am J Respir Crit Care Med 2004; 169: 39-45.

27 Clozel M, Hess P, Rey M, et al. Bosentan, sildenafil, and their combination in the monocrotaline model of pulmonary hypertension in rats. Exp Biol Med (Maywood) 2006; 231: 967-973.

28 Andersen A, Nielsen JM, Peters CD, et al. Effects of phosphodiesterase-5 inhibition by sildenafil in the pressure overloaded right heart. Eur J Heart Fail 2008; 10: 1158-1165.

29 Tawara S, Fukumoto Y, Shimokawa H. Effects of combined therapy with a Rho-kinase inhibitor and prostacyclin on monocrotalineinduced pulmonary hypertension in rats. J Cardiovasc Pharmacol 2007; 50: 195-200.

30 Fukumoto Y, Matoba T, Ito A. Acute vasodilator effects of a Rhokinase inhibitor, fasudil, in patients with severe pulmonary hypertension. Heart 2005; 91: 391-392.

31 Benza RL, Park MH, Keogh A, et al. Management of pulmonary arterial hypertension with a focus on combination therapies J Heart Lung Transplant 2007; 26: 437-446.

32 Mathai SC, Girgis RE, Fisher MR, et al. Addition of sildenafil to bosentan monotherapy in pulmonary arterial hypertension. Eur Respir J 2007; 29: 469-475.

33 Oka M, Fagan KA, Jones PL, et al. Therapeutic potential of RhoA/ Rho kinase inhibitors in pulmonary hypertension. $\mathrm{Br}$ J Pharmacol 2008; 155: 444-454.

34 Weigand L, Sylvester JT, Shimoda LA. Mechanisms of endothelin-1induced contraction in pulmonary arteries from chronically hypoxic rats. Am J Physiol Lung Cell Mol Physiol 2006; 290: L284-L290.

35 Takemoto M, Sun J, Hiroki J, et al. Rho-kinase mediates hypoxiainduced downregulation of endothelial nitric oxide synthase. Circulation 2002; 106: 57-62.

36 Mita S, Kobayashi N, Yoshida K, et al. Cardioprotective mechanisms of Rho-kinase inhibition associated with eNOS and oxidative stress-LOX-1 pathway in Dahl salt-sensitive hypertensive rats. J Hypertens 2005; 23: 87-96.

37 Zhang J, Li XX, Bian HJ, et al. Inhibition of the activity of Rhokinase reduces cardiomyocyte apoptosis in heart ischemia/ reperfusion via suppressing JNK-mediated AIF translocation. Clin Chim Acta 2009; 401: 76-80.

38 Chien R. Signaling mechanisms for the activation of an embryonic gene program during the hypertrophy of cardiac ventricular muscle. Basic Res Cardiol 1992; 87: Suppl. 2, 49-58.

39 Rajabi M, Kassiotis C, Razeghi P, et al. Return to the fetal gene program protects the stressed heart: a strong hypothesis. Heart Fail Rev 2007; 12: 331-343. 